\title{
A STAGGERED MATERIAL POINT METHOD AND FINITE ELEMENT METHOD COUPLING SCHEME USING GAUSS SEIDEL COMMUNICATION PATTERN
}

\author{
VERONIKA SINGER* ${ }^{*}$ BODHINANDA CHANDRA ${ }^{\dagger}$, ANTONIA \\ LARESE $^{\dagger \dagger}$ AND ROLAND WÜCHNER* \\ *Department of Civil, Geo and Environmental Engineering \\ Technical University of Munich (TUM) \\ Arcisstraße 21, 80333 Munich, Germany \\ e-mail: veronika.singer@tum.de, wuechner@tum.de, web page: http://www.bgu.tum.de/st \\ ${ }^{\dagger}$ Department of Civil and Environmental Engineering \\ University of Carlifonia \\ Berkeley, CA, 94720, United States \\ e-mail:bchandra@berkeley.edu \\ ${ }^{\dagger \dagger}$ Department of Mathematics "Tullio Levi Civita" \\ Universitá degli Studi di Padova \\ Padova 35121, Italy \\ e-mail: antonia.larese@unipd.it
}

Key words: Soil-Structure Interaction, Staggered Coupling, Material Point Method, Finite Element Method, Boundary Conditions

\begin{abstract}
The goal of the present work is the simulation mass movement hazards, involving fast and large soil deformation, interacting with flexible protection structures. For the simulation of those large deformation phenomena, involving complex history dependent material laws, the Material Point Method (MPM) is a powerful method, as the particles move trough a fixed background mesh. This allows to overcome the classical limitation of the Finite Element Method (FEM) related to mesh distortion in large strain problems. Therefore, a staggered or partitioned coupling scheme is proposed, combining the advantages of FEM and MPM by solving both models separately using their respective established environment, whereas the communication between the two fields is achieved by mapping boundary conditions on the shared interface. In this work a Gauss-Seidel communication pattern is considered, leading to the necessity of imposing Dirichlet Boundary Conditions on one interface (in this study: FEM) and Neumann Boundary Conditions on the corresponding counterpart (in this study: MPM). For validation purposes, a structural example with analytical solution is chosen.
\end{abstract}




\section{INTRODUCTION}

Mass movements hazards involving fast and large soil deformation have increased significantly in the past decades due to the climate change and global warming. Those phenomena, avalanches and mud-flow, can cause extensive damage on landscapes and infrastructures. Therefore, further assessment and prediction on the effects on such disasters and countermeasures are in high social and economic demands.

For the simulation of solid mechanics problems, including history dependent material laws and large deformations, MPM has gained a remarkably increasing popularity in the last years. As the material domain is discretized by Lagrangian moving particles, whereas the governing equations are solved at a stationary background grid, mesh-distortion problems are eliminated. This method was first introduced by $[2,3]$ as an extension of Particle-InCell (PIC) method [4] whereas the implicit version of the method, utilized in this study was introduced by [5].

Although MPM has been proven, to work robustly for problems involving large deformation materials, the accuracy of the integration done in the particle integration is significantly lower than the Gaussian quadrature, which is used in traditional FEM. Furthermore, the cell-crossing error and numerical fracture can lead to less accurate results compared to FEM, which is a very efficient method for structural dynamics. Therefore

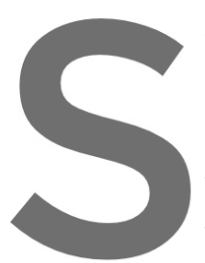
especially for soil-strut MPM and take the Therefore, a staggered the MPM and the FE whereas the communic
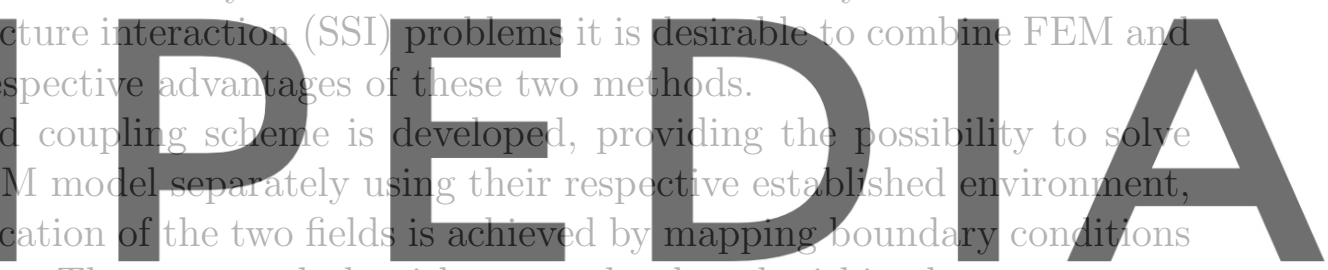

on the shared interface. The presented algorithms are developed within the open source

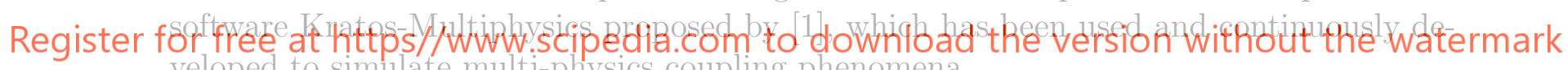
veloped to simulate multi-physics coupling phenomena.

\section{GOVERNING EQUATIONS}

\subsection{Strong Form}

The governing equations, i.e. the mass and momentum conservation equations, for a body $\mathcal{B}$, which occupies a domain $\Omega$ in a three-dimensional Euclidean space $\mathcal{E}$ can be written as:

$$
\begin{array}{ll}
\frac{d \rho}{d t}+\rho \nabla \cdot \dot{\mathbf{u}}=0 & \text { in } \Omega \\
\rho \ddot{\mathbf{u}}=\nabla \cdot \sigma+\rho \mathbf{b} & \text { in } \Omega
\end{array}
$$

where $\rho$ is the mass density, $\mathbf{b}$ is the volume acceleration and $\sigma$ is the symmetric Cauchy stress tensor. The first and second material derivative of the displacement $\mathbf{u}$ are the velocity and the acceleration, respectively. Resulting from the time-dependent map $\mathbf{\Phi}(\mathbf{X}, t)$, the displacement is expressed by the difference between the current configuration $\mathbf{x}$ and the reference configuration $\mathbf{X}$ which describes the kinematic relation for each point within 
the body $\mathcal{B}$ and is illustrated in Figure 1.

$$
\mathbf{u}(t)=\mathbf{x}(t)-\mathbf{X}
$$

The problem 1 - 2 is fully defined with the boundary conditions

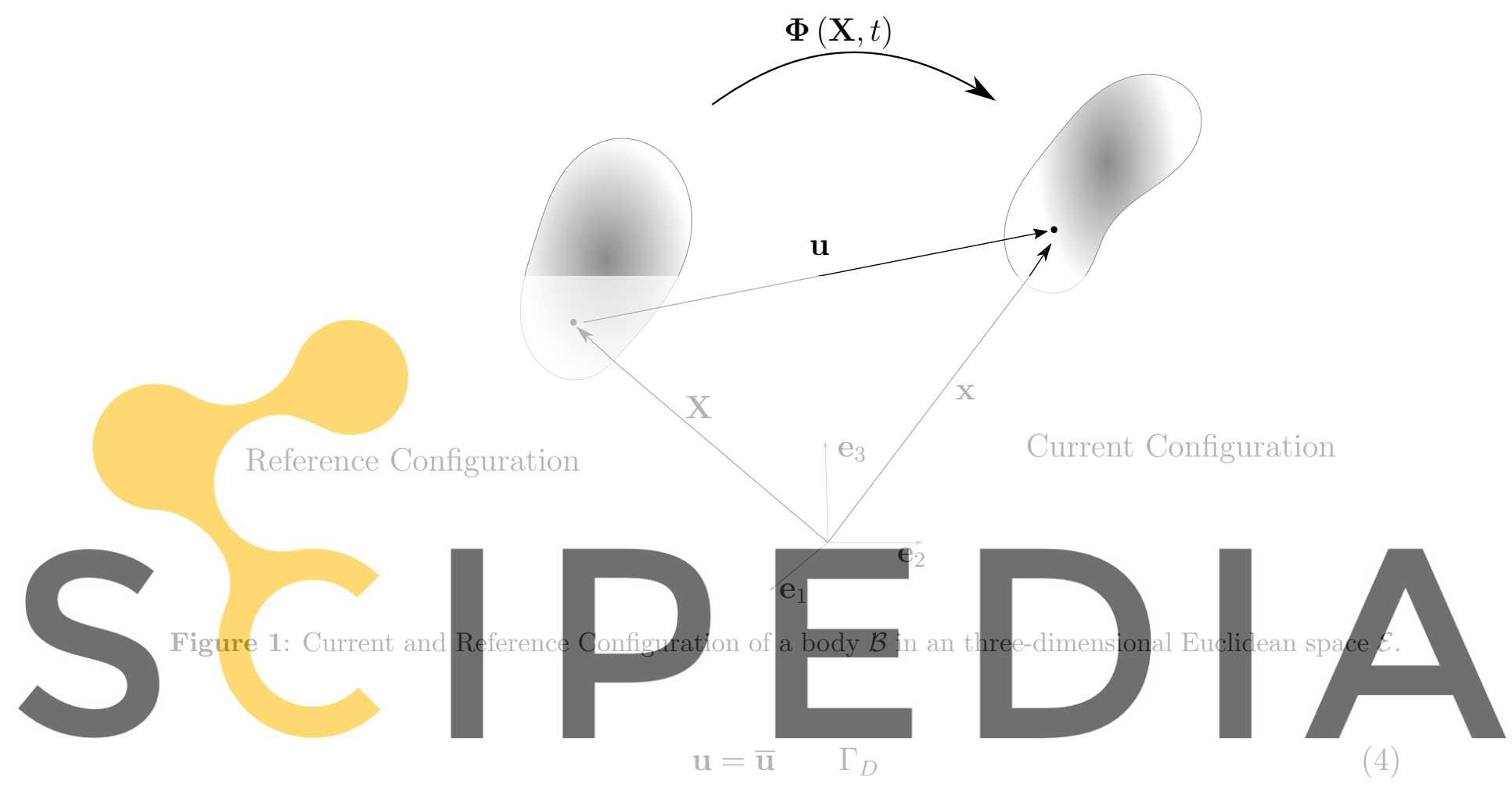
Register for free at https//www.scipedia.com $m_{\mathrm{n}}$ to down $_{\mathrm{t}}{ }_{N}$ ad the version without the watermark

where $\overline{\mathrm{u}}$ is a prescribed displacement on the Dirichlet boundary $\Gamma_{D}$ and $\overline{\mathrm{t}}$ is a traction vector on the Neumann boundary $\Gamma_{N}$.

\section{$2.2 \quad$ Virtual Work}

Multiplying the momentum equation with a virtual displacement field $\delta \mathbf{u}$ and integrating over the domain $\Omega \subset \mathcal{E}$ leads to the weak form of the balance equation

$$
\delta W=\delta W_{i n t}-\delta W_{e x t}+\delta W_{k i n}=0
$$

with the internal work contribution, obtained by partial integration, the external and the kinetic work

$$
\begin{gathered}
\delta W_{\text {int }}:=\int_{\Omega} \sigma: \delta e \mathrm{~d} \Omega \\
\delta W_{\text {ext }}:=\int_{\Omega} \rho \mathbf{b} \cdot \delta \mathbf{u} \mathrm{d} \Omega+\int_{\Gamma_{N}} \sigma \mathbf{n} \cdot \delta \mathbf{u} \mathrm{d} \Gamma_{N}
\end{gathered}
$$




$$
\delta W_{k i n}:=\int_{\Omega} \rho \ddot{\mathbf{u}} \cdot \delta \mathbf{u d} \Omega
$$

respectively. Equation 6 can be expressed through the variation of the virtual work w.r.t. $\delta \mathbf{u}$ as

$$
\delta W=\frac{\partial W}{\partial \mathbf{u}} \delta \mathbf{u}=0
$$

Since, in the current work, geometric and material nonlinearities are considered, a linearization of the weak form is necessary, and thus, the Newton-Raphson method, which is based on Taylor's theorem, is used to approximate the solution iteratively.

\subsection{Discretization in Space and Time}

In FEM the body $\mathcal{B}$ is discretized into a finite number $n_{e}$ of non-overlapping subdomains $\Omega_{e}$

$$
\mathcal{B} \approx \mathcal{B}^{h}=\bigcup_{e=1}^{n_{e}} \Omega_{e}
$$

where the subscript $\mathrm{h}$ is used to indicate the approximative character. Therefore the continuous fields are approximated by discrete quantities in the nodes and locally confined basis functions. This
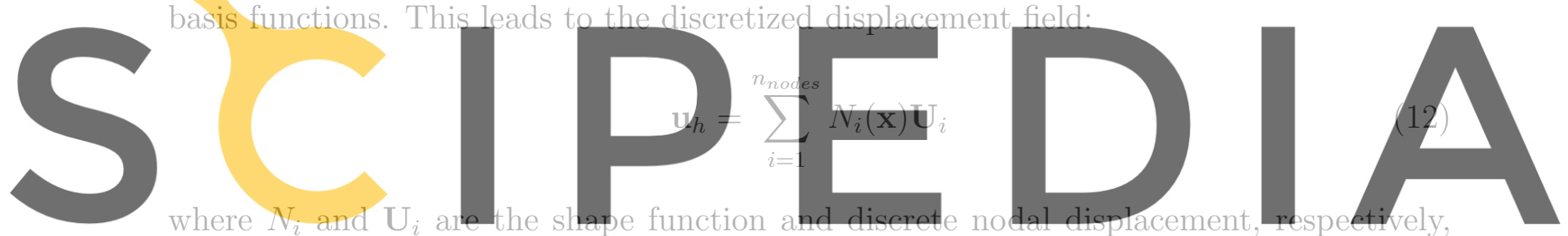

corresponding to node $i$ of the element, which in turn consists of nnodes nodes. The same

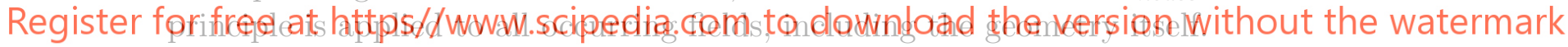

Considering the time derivatives, an implicit time integration scheme of the Newmark-

beta method is utilized. Hence equation 10 transforms to:

$$
\delta W=\frac{\partial W}{\partial \mathbf{u}_{h}} \delta \mathbf{u}_{h}=-\mathbf{R} \cdot \delta \mathbf{u}_{h}=0
$$

meaning, that the residual force vector $\mathbf{R}$ has to vanish, as the virtual displacements $\delta \mathbf{u}_{h}$ are arbitrary. Since material and geometric nonlinearities are considered, an iterative solution approach namely the Newton Raphson algorithm is applied, which introduces the tangential stiffness matrix $\mathbf{K}$ as a linearization of the residual vector $\mathbf{R}$ :

$$
\operatorname{LIN}(\mathbf{R})=\mathbf{R}+\frac{\partial \mathbf{R}}{\partial \mathbf{u}_{h}} \Delta \mathbf{u}_{h}=\mathbf{R}+\mathbf{K} \Delta \mathbf{u}_{h}=0
$$

Also in MPM, the continuous fields in MPM are approximated by discrete nodal values 
and the corresponding shape functions, by introducing a computational background grid. The body $\mathcal{B}$, however, is discretized into $n_{p}$ Lagrangian moving material points as:

$$
\mathcal{B} \approx \mathcal{B}^{h}=\bigcup_{p=1}^{n_{p}} \Omega_{p}
$$

with a finite volume of the body $\Omega_{p}$. Those material points carry the history dependent variables and material information during the calculation process, whereas the background grid is reset after each time-step. Therefore this leads to the necessity of inter- and extrapolating data from the material points to the nodes of the computational background grid and vice versa, resulting into the three phases of a MPM calculation:

1. Initialization phase: A search is performed, to define the background grid element, which belongs to each material point, before the necessary variables are mapped via mass projection to the corresponding nodes as initial conditions.

2. Lagrangian phase: Solution of the discretized governing equations. Coincides with the solution step for an Updated Lagrangian Element in FEM.
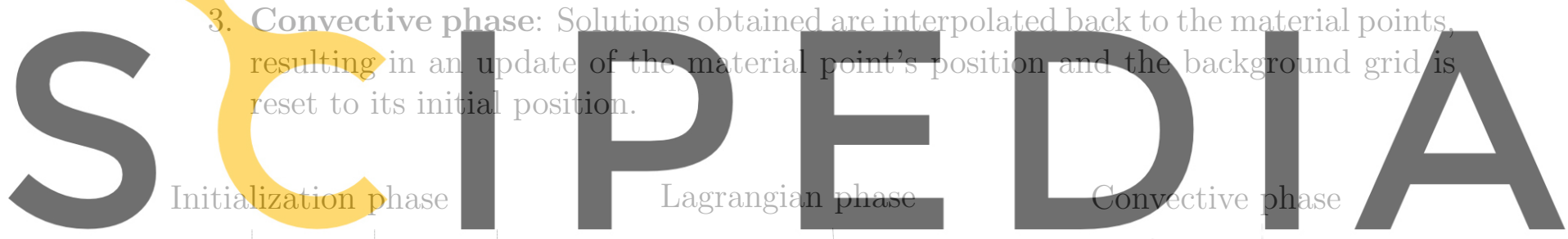

Register for free at https//wyw.scipedia.com to.download the version without the watermark

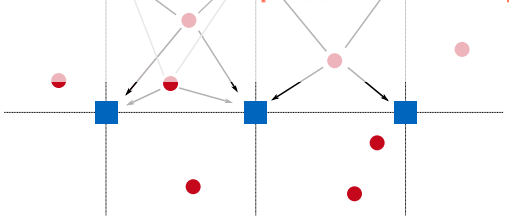

grid nodes
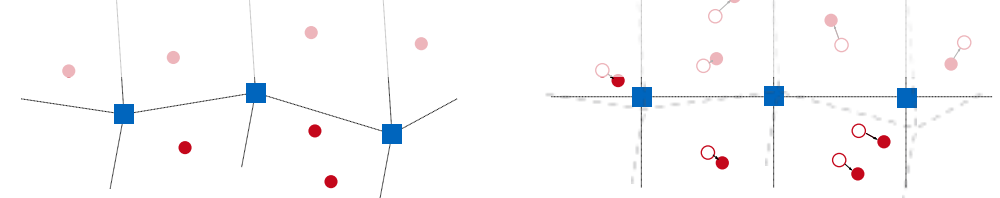

- material points o. material point update

Figure 2: (a)Initialization phase, (b) Lagrangian phase and (c) Convective phase. Square markers identify the grid nodes while round markers indicate the material points.

which is also illustrated in figure 2 .

For both methods, the integrals in the discretized equations 14 have to be solved. While in Lagrangian FEM usually Gauss integration is used, as the elements deform according to the body, "particle" integration is applied in MPM.

As MPM allows to overcome the the classical limitation of FEM related to mesh distortion in large strain problems, a staggered coupling scheme is introduced, to solve the 
engineering problem of large mass movement hazards interacting with flexible protection structures. Therefore the communication of the two separated fields is shifted to the shared interface leading to the necessity of imposing boundary conditions on each interface. While the imposition of boundary conditions is straightforward in FEM, special attention should be made in MPM, which is introduced in the next section.

\subsection{Boundary Conditions in MPM}

As in MPM the particles move independently of the computational background grid, the boundaries $\Gamma$, both, the Neumann $\Gamma_{N}$ and Dirichlet $\Gamma_{D}$ boundaries, usually do not coincide with the background grid nodes. In Lagrangian FEM, as the mesh moves according to the body deformation, the boundary conditions can be applied directly at the nodes, the so calles grid-conforming boundaries. Also in MPM, some strategies have been introduced, to achieve grid-conforming boundary imposition, just mentioning, the moving grid approach [8]. However, regarding the coupling of MPM with FEM or other physics, the robust imposition of non-conforming boundary conditions is crucial. Following the concept of MPM, a particle discretization is applied, to represent the continuous boundary $\Gamma$ with a discrete number of boundary particles $b$ leading to the approximation:

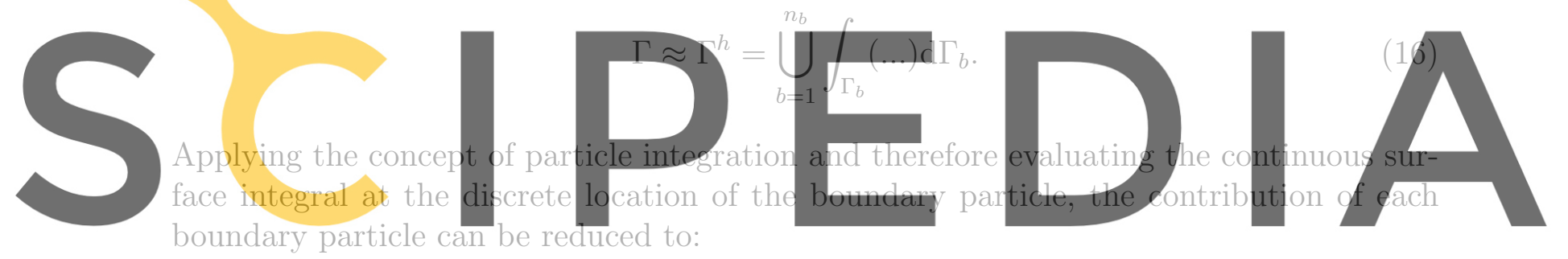

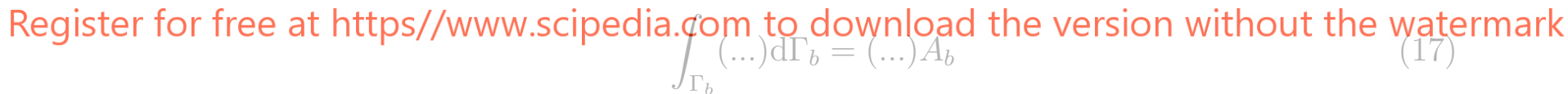

assuming, that the quantities inside the surface integral are constant within the surface. Those mass-less boundary particles track the deformation of the interface and carry the respective information for the boundary imposition.

For the imposition of Dirichlet Boundary conditions a penalty augmentation is a suitable method and its accuracy has been proven in [10]. Using those introduced boundary particles, moving boundaries, slip boundaries as well as releasing-contact conditions can be realized, by penalty augmentation. Furthermore, this boundary imposition can also be used for a staggered coupling approach with FEM, as shown in [11]. Further verification and validation tests are planned to be performed for this coupling scheme in near future, but are out of scope of the current work.

In the case of Neumann boundaries, the particles are carrying the value of the Point Load and the corresponding position of the particle. During the calculation process, 
the Point Load $P_{b}$ is mapped via the shape functions to the corresponding nodes of the computational background grid.

$$
P_{i}=N_{i}\left(x_{b}\right) \cdot P_{b}
$$

where $P_{i}$ is the resulting value at the node $i$ of the background grid and $N_{i}\left(x_{b}\right)$ is the corresponding shape function evaluated at the point-load position $x_{b}$.

Special treatment has to be done for elements which contain boundary particles but no material points. Therefore, only the nodes of the background grid are considered which also carry a mass resulting from the material points and ensuring the interaction with the material. Therefore the considered shape functions are modified accordingly to fulfill unity on the one hand side and preserve the sum of forces on the other hand side.

\section{COUPLING OF MPM AND FEM}

In this study a partitioned or staggered approach is considered which keeps the solution of the two subsystems, structural domain $\Omega_{S}$ with boundary $\Gamma_{S}$ and the particle domain $\Omega_{P}$ with boundary $\Gamma_{P}$, independently of each other. The communication between the subsystems is shifted to the shared interface
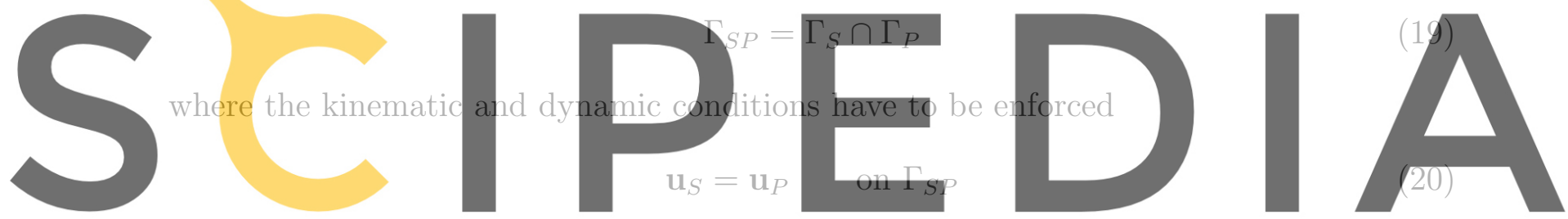

$$
\overline{\mathrm{t}}_{S}=\overline{\mathrm{t}}_{P}
$$

on $\Gamma_{S P}$

(21)

Register for free at https//www.scipedia.com to download the version without the watermark where $\mathbf{u}_{S}$ and $\mathbf{u}_{p}$ are the displacements of the structure and the particles and $\mathbf{t}_{S}$ and $\mathbf{t}_{P}$ are their traction, respectively. The kinematic constraint makes sure, that both domains deform consistently at the interface without gaps and overlaps, whereas the dynamic condition specifies the load balance at the shared interface. Due to the independent discretization of each domain, the Nearest Neighbor mapping technique [6] is applied to enforce the respective conditions at non-matching grids.

In the current work the classical Dirichlet-Neumann decomposition approach is applied, meaning, that reaction forces, which are the resultants of the traction multiplied by the respective area of the discretized surface, are transferred from the FEM interface to MPM and displacements and velocity are transferred back from MPM as prescribed displacements in FEM resulting in the communication pattern, depicted in Figure 3. Depending on the simulation to be performed, it is possible to start either with MPM and therefore mapping first the displacement to FEM as prescribed displacement and receiving the respective Reaction Forces as Point Loads or, alternatively, starting with the FEM calculation and therefore sending the Reaction Forces first to MPM and receiving the displacement as an answer. 


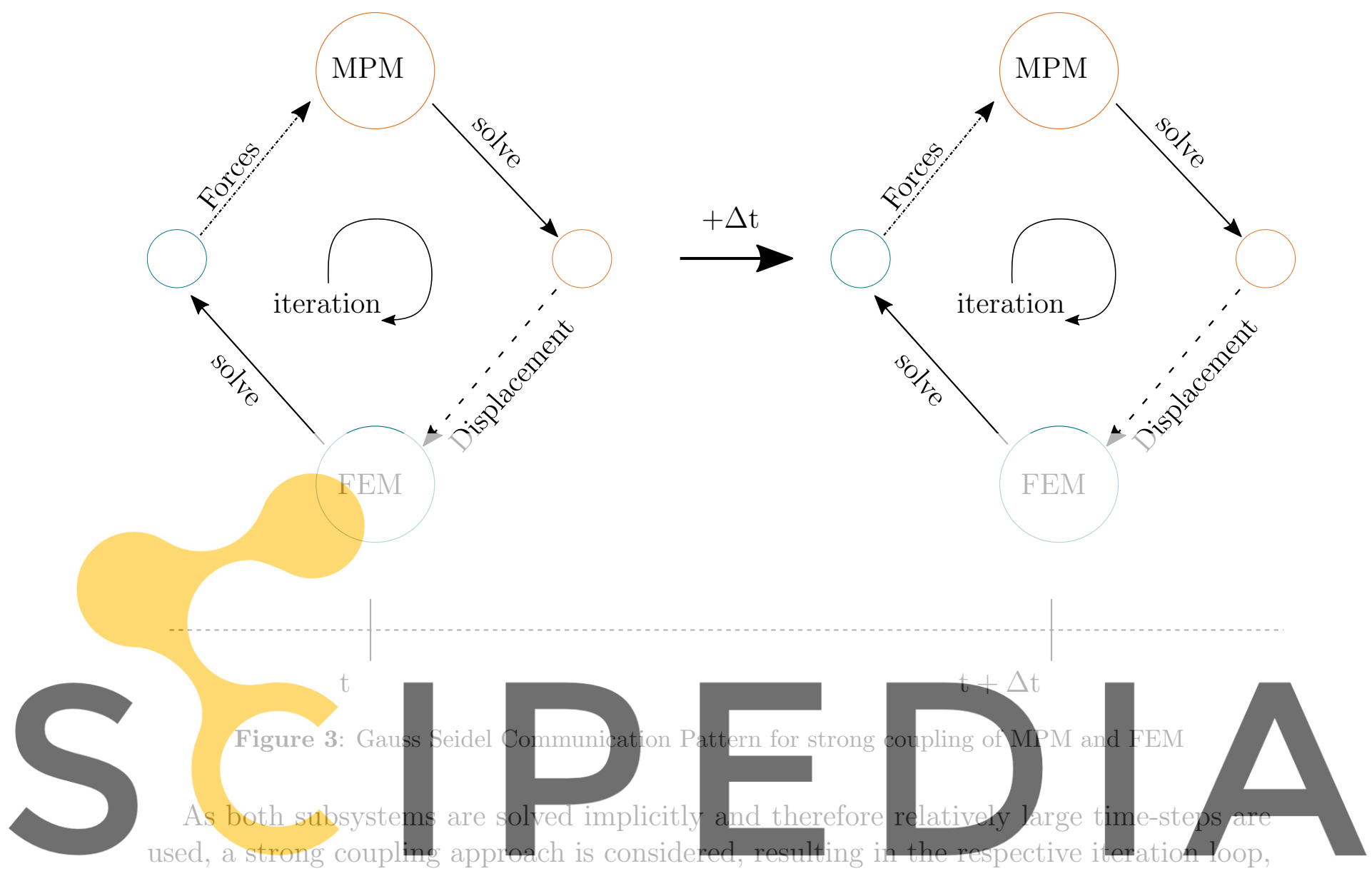

until the residual criteria is fulfilled. Furthermore, large time steps typically lead to large

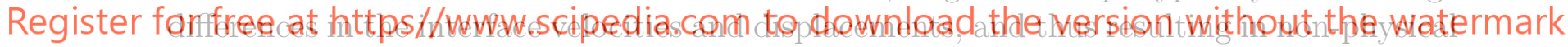

reaction forces. To overcome this problem, convergence-accelerators [9] can be used, which means, that the transferred data is applied gradually.

\section{Numerical Example}

The first step, before running challenging large scale examples, as the interaction of landslides with protection structures, is the validation for the coupling algorithm and the mapping parameters. Therefore, validation tests are performed, and compared to the analytical solution. Therefore, as a first example, a static, linear elastic beam, clamped on both sides, under self-weight with a total length of $\mathrm{L}=8 \mathrm{~m}$ and a square crosssection with unit area of $\mathrm{A}=1 \times 1 \mathrm{~m}^{2}$ is considered. The material parameters are set as follows: density $\rho=1000 \mathrm{~kg} / \mathrm{m}^{3}$, Young's modulus $\mathrm{E}=90 \mathrm{MPa}$ and Poisson's ratio $\nu=0.0$. The sketch of the system can be found in Figure 4 including a detailed sketch of the shared interface. The left hand side of the beam is modeled using MPM whereas for the right hand side FEM is used. For the discretization of the FEM model, a structured, quadrilateral mesh is considered with an element size of $0.04 \mathrm{~m}$. Also for 
Point A

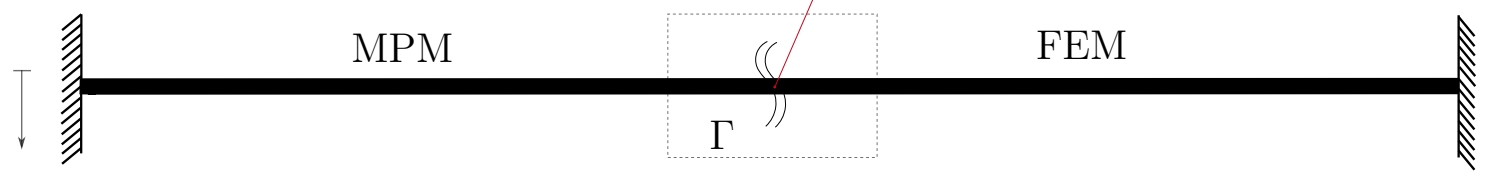

gravity: $9.81 \frac{\mathrm{N}}{\mathrm{m}^{2}}$

$8 \mathrm{~m}$
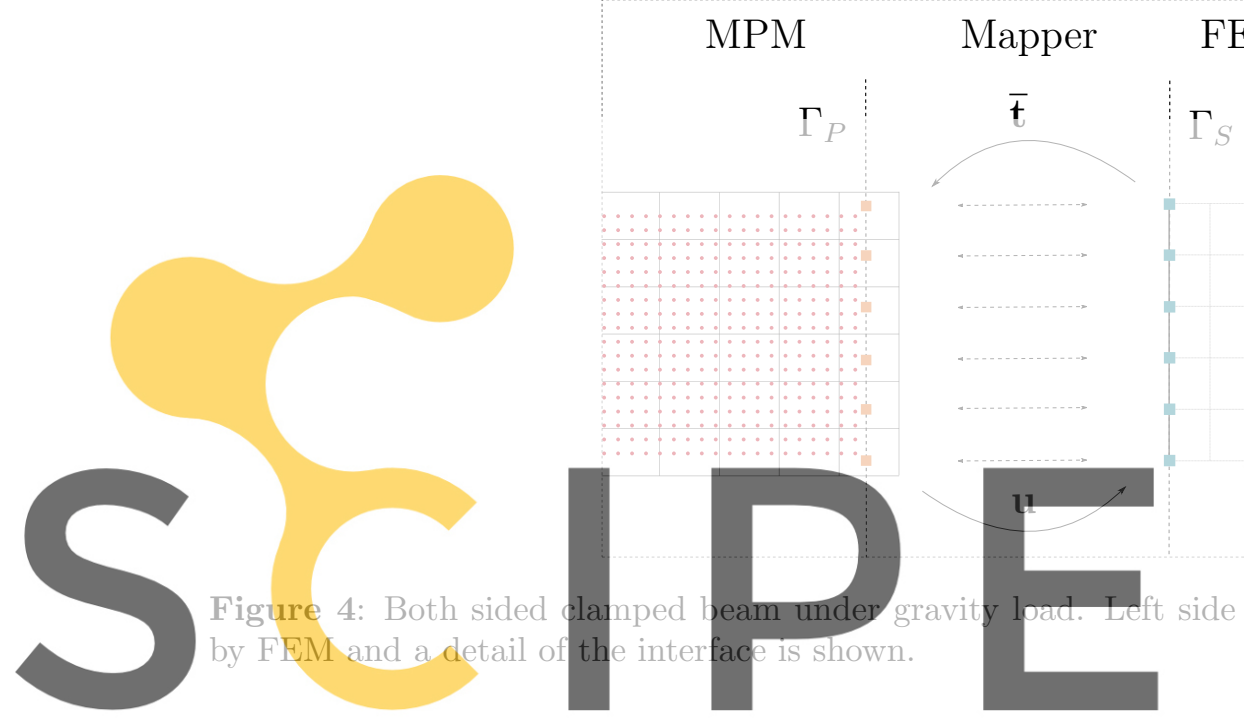

FEM
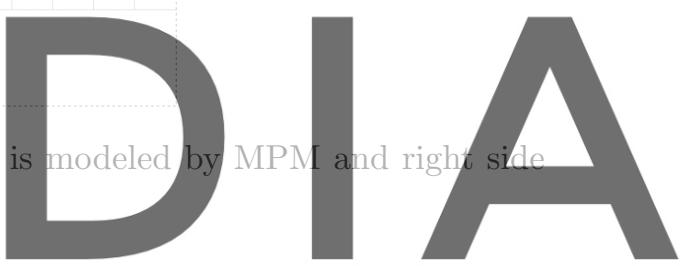

MPM, a quadrilateral background grid with mesh size of $0.04 \mathrm{~m}$ is assumed, whereas

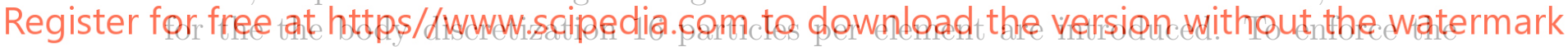

respective boundary conditions, a conforming Dirichlet condition is considered at the

nodes of the FEM grid at the shared interface $\Gamma_{S}$. For the MPMi counterpart Neumann $\Gamma_{P}$ boundary particles are introduced, which are able to impose the respective reaction forces from FEM as Point Loads to MPM and are located independently of the considered background grid. To eliminate a possible mapping error for this example, the boundary particles have the same coordinates as the respective nodes of the FEM counterpart. Therefore the mapping reduces to a copy operation with a switch of the sign. In the near future, also other mapping techniques, which for example can be found in [7], will be investigated.

For this example, the vertical displacement of the beam at the evaluation Point $A$, which is the center of this structure, should be compared to the analytical solution, which can be calculated by:

$$
w_{A}^{r e f}=\frac{g \cdot \rho \cdot A \cdot L^{4}}{384 \cdot E I}+\frac{g \cdot \rho \cdot A \cdot L^{2}}{8 \cdot G A_{s}}
$$


where $I=b h^{3} / 12$ is the inertia of the beam section, $A_{s}=5 / 6 \mathrm{~A}$ is the reduced cross-section area due to the shear effect and $G$ is the shear modulus, which is equal to $G=\frac{E}{2(1+\nu)}$. Figure 5 shows the final deformation of the coupled beam model under gravity load and
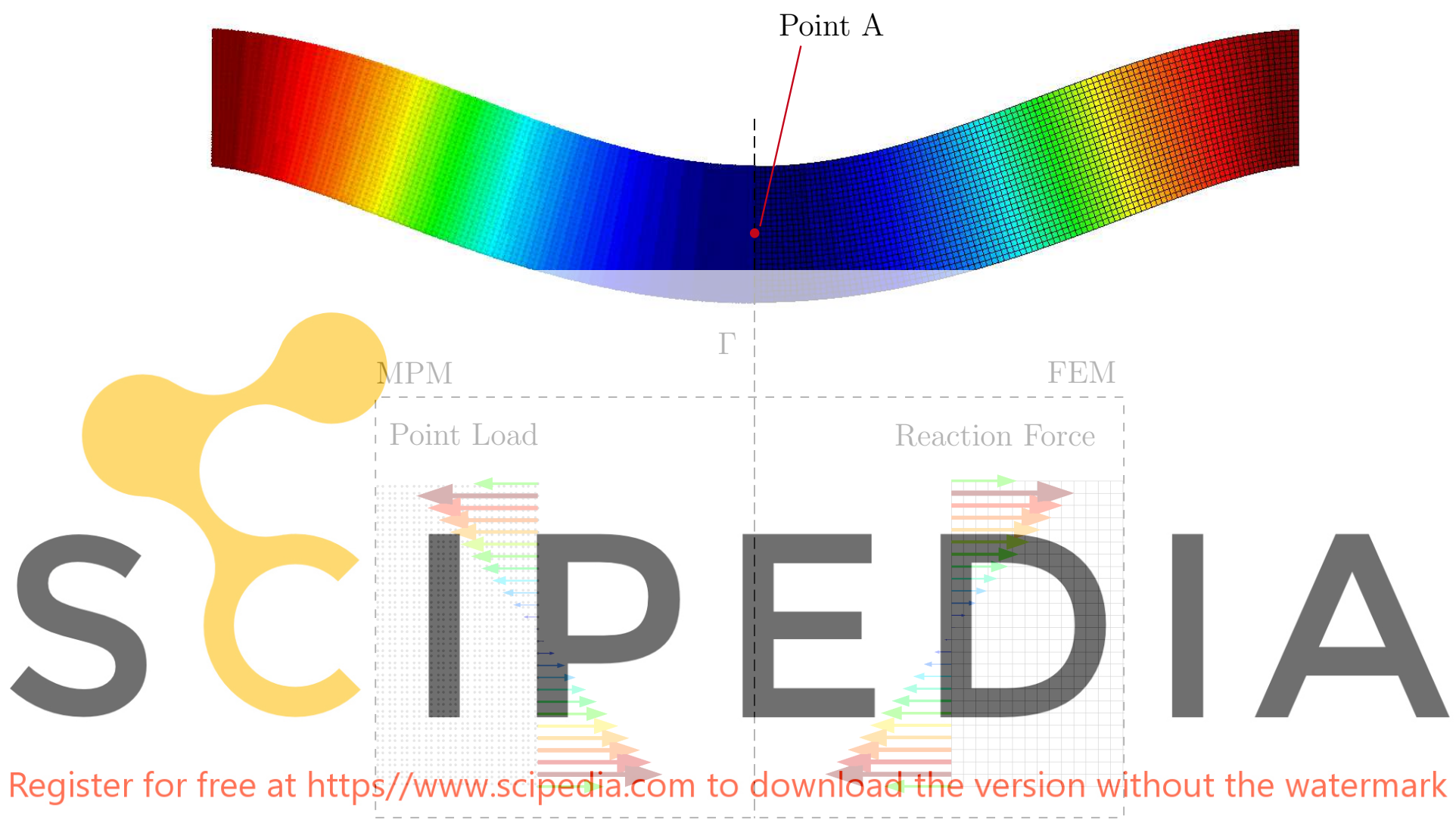

Figure 5: Final deformation of the both sided clamped beam under gravity load using the proposed staggered approach and the resulting forces at the shared interface.

the resulting displacement at Point $A$ is $\mathrm{w}_{\mathrm{A}}=0.0160109 \mathrm{~m}$. Compared to the analytical result $\mathrm{w}_{A}^{\text {ref }}=0.0160448$ this leads to an relative error of $e_{r}=\left|\frac{w_{A}-w_{A}^{r e f}}{w_{A}^{r e f}}\right|=0.21 \%$ and an absolute error of $e_{a}=\left|w_{A}-w_{A}^{r e f}\right|=0.0034 \%$.

This result could even be improved, by decreasing the grid size, as the distribution of the Point Load over the background element has a main effect on the final result. If the calculation is repeated with half the grid size, meaning an element size of 0.02 for each, the FEM and MPM part, the relative error reduces to:

$$
e_{r}=\left|\frac{w_{A}-w_{A}^{r e f}}{w_{A}^{r e f}}\right|==\frac{0.0160172-0.0160448}{0.0160448}=0.17 \% .
$$


The continuity of the displacements is proven by the resulting deformation of the considered example. But also the traction at the shared interface is mapped perfectly, as can be seen in the lower part of Figure 5. The Reaction Forces at the interface $\Gamma_{S}$, which are the resultants of the traction multiplied by the respective area of the discretized interface, are mapped by the nearest neighbor mapper to the MPM interface $\Gamma_{P}$ as Point Loads with opposite sign. As the location of the introduced boundary particles in MPM coincide with the coordinates of the FEM interface nodes, no mapping error is introduced in this example, resulting in equivalent values for the forces on both sides of the interface. Due to the symmetry of the considered example, the resulting forces are horizontal and sum up to the final bending moment of the beam formulation $\mathrm{M}=26,15 \mathrm{kNm}$ at the interface with a relative error of $e_{r}=0.049 \%$ compared to the analytical solution:

$$
\mathrm{M}^{\mathrm{ref}}=\frac{g \cdot \rho \cdot A \cdot L^{2}}{24}=26,16 \mathrm{kNm} .
$$

This example proves the applicability of the proposed staggerd coupling scheme for static cases. Further verification and validation tests, including dynamic effects, are planned to be performed in the near future.

\section{CONCLUSIONS}

A staggered strong coupling scheme is presented, to couple implicit MPM and FEM. Utilizing this proposed approach one can benefit of the strengths of both methods for the simulation of landslides interacting with protection structures, as FEM efficiently leads to accurate results for structural dynamics, whereas MPM is preferable for the simulation of large and non-linear soil deformation. Nevertheless, some future works are necessary to do some verification and validation tests, including dynamic effects on the one hand side and adapt the proposed scheme to real-scale landslide hazards involving complex multi-phase flows of particles with different size on the other hand side.

\section{REFERENCES}

[1] Dadvand, P., Rossi, R. and Oñate, E. An object-oriented environment for developing finite element codes for multi-disciplinary applications. Archives of computational methods in engineering, 17(3):253297.

[2] Sulsky, D., Chen, Z. and Schreyer, H.L. A particle method for history-dependent materials. Computer methods in applied mechanics and engineering (1994) 118(12):179196.

[3] Sulsky, D., Zhou, S.-J., and Schreyer, H.L. Application of a particle-in-cell method to solid mechanics. Computer physics communications (1995) 87(1-2):236252.

[4] Harlow, F.H., The particle-in-cell computing method for fluid dynamics. Methods Comput. Phys. (1964) 3:319343. 
[5] Iaconeta, I., Larese, A., Rossi, R. and Oñate, E. Comparison of a material point method and a galerkin meshfree method for non-linear incompressible solid mechanics. Computational Mechanics (2017) pages 1-18.

[6] Bucher, P., Development and implementation of a parallel framework for nonmatching grid mapping. Master thesis, Technical University Munich

[7] Gatzhammer, B., Efficient and Flexible Partitioned Simulation of Fluid-Structure Interactions. Ph.D. thesis, Technical University Munich(2014)

[8] Kafaji, I. K., Formulation of a dynamic material point method (MPM) for geomechanical problems. Ph.D. thesis, University of Stuttgart(2013)

[9] Bogaers, A. E., Kok, S., Reddy, B. D. and Franz T. Quasi-newton methods for implicit black-box FSI coupling. Computer Methods in Applied Mechanics and Engineering (2014) 279:113132

[10] Chandra, B., Singer, V., Teschemacher, T., Wüchner, R. and Larese, A. Nonconforming Dirichlet boundary conditions in implicit material point method by means of penalty augmentation. Acta Geotechnica(2021)

[11] Chandra, B., Larese, A., Bucher, P. and Wüchner, R. Coupled soil-structure interaction modeling and simulation of landslide protective structures. VIII International Conference on Computational Methods for Coupled Problems in Science and Engineering (2019) 